\title{
BMJ Open A novel inpatient complex pain team: protocol for a mixed-methods evaluation of a single-centre pilot study
}

\author{
Luke Mordecai, ${ }^{1}$ Cecilia Vindrola-Padros, ${ }^{2}$ Victoria J Wood, ${ }^{2}$ Nicholas Swart, ${ }^{2}$ \\ Stephen Morris, ${ }^{2}$ Amanda Williams, ${ }^{3}$ Natasha Curran, ${ }^{1}$ Ramani Moonesinghe ${ }^{4}$
}

To cite: Mordecai L, VindrolaPadros C, Wood VJ, et al. A novel inpatient complex pain team: protocol for a mixedmethods evaluation of a singlecentre pilot study. BMJ Open 2018;8:e019058. doi:10.1136/ bmjopen-2017-019058

- Prepublication history for this paper is available online. To view these files, please visit the journal online (http://dx.doi. org/10.1136/bmjopen-2017019058).

Received 13 August 2017 Revised 18 December 2017 Accepted 22 December 2017

Check for updates

${ }^{1}$ Pain Management Centre, University College London Hospital, London, UK ${ }^{2}$ Department of Applied Health Research, University College London, London, UK

${ }^{3}$ Research Department of Clinical, Educational and Health Psychology, University College London, London, UK

${ }^{4}$ NIAA Health Services Research Centre, Royal College of Anaesthetists, London, UK

Correspondence to

Dr Luke Mordecai;

luke.mordecai@gmail.com

\section{ABSTRACT}

Introduction Complex pain is a debilitating condition that is responsible for low quality of life and significant economic impacts. Although best practice in the treatment of complex pain employs a multidisciplinary team, many patients do not have access to this care, leading to poor outcomes.

Methods and analysis This study evaluates a novel inpatient complex pain team at a large London teaching hospital. A multidisciplinary pain team comprising specialist doctors, nurses, psychologists and physiotherapists was instituted for inpatients with complex pain who will undergo an intense and bespoke evidencebased programme which will then be integrated into the community. A mixed-methods evaluation will take place and patients will be recruited over the course of 1 year. A qualitative arm will interview 15 staff and 15 patients on recruitment and again 6 months later looking to compare and contrast the new pain team with past experiences of pain management. A quantitative arm will assess clinical outcomes using validated scoring tools. An economic evaluation will seek to evaluate the relative cost of the service by comparing healthcare costs before and after the intervention.

Ethics and dissemination The study was categorised as a service evaluation, so formal ethical approval was not considered necessary. Participant recruitment began in January 2016 and the 1-year follow-up will end in November 2017. The results of this study will be published in 2018.

\section{INTRODUCTION}

The profile of pain and its impact on individuals' quality of life, the health system and the economy is increasingly recognised. ${ }^{1}$ In 2008, the Chief Medical Officer for England featured pain as one of five special topics in his Annual Report. ${ }^{1}$ He concluded that chronic pain and its consequences were poorly controlled and identified it as a substantial public health problem affecting all ages. Only $14 \%$ of sufferers had ever seen a pain specialist and estimated cost to the Exchequer of back pain alone was $£ 12.3$ billion per annum. ${ }^{1}$ Large healthcare spends are corroborated elsewhere in the literature where

\section{Strengths and limitations of this study}

- Use of a comprehensive mixed methodology to provide a detailed evaluation of a novel inpatient team from multiple different perspectives.

- Multiple cohorts are used for comparisons in an attempt to offset the lack of a true control group.

- Small, non-randomised study population means further work is required to provide absolutely robust conclusions.

- Greater than 6-month follow-up time would ideally be required to make a full assessment of the longterm economic and clinical outcomes. This is prevented by funding and time constraints.

primary care management of patients with chronic pain was estimated to account for 4.6 million appointments per year; at a cost of $£ 69$ million. 2

In 2011, The Health Survey for England confirmed the magnitude of the problem and stated that $31 \%$ of men and $37 \%$ of women met the criteria for a diagnosis of chronic pain ${ }^{3}$ and larger incidence was noted with increasing age and lower socioeconomic status. This report also detailed the association between chronic pain and other illnesses, especially anxiety and depression, although the direction of causality remains unknown.

In 2011 and 2012, The National Pain Audit was carried which involved all National Health Service (NHS) Trusts and Primary Care Trusts capturing data from all specialist pain clinics within primary and secondary care in England. It reported that $20 \%$ of those surveyed had attended an Emergency Department within the last 6 months, and $66 \%$ had made three or more presentations to healthcare providers over the same period. ${ }^{4}$ Access to the gold standard multidisciplinary specialist clinics, as defined by the presence of a specialist doctor, physiotherapist and psychologist, was poor with $60 \%$ of clinics not meeting the latter criteria. 
Further evidence confirms the high incidence of chronic pain in the UK, which is now thought to affect up to half of the population, and, given an ageing demographic, this will only increase. ${ }^{5}$ Other evidence suggests that it is a global issue with figures from the USA estimating that chronic pain affects $10 \%-20 \%$ of the general population $^{6-8}$ resulting in an economic burden in excess of $\$ 70$ billion annually. ${ }^{9}$

Inadequately treated pain escalates, causing high degrees of distress and disability and increases the burden of care across all healthcare services. Additionally, the overall quality of life for people with chronic pain is very poor, averaging a life score of 0.4 where 1 is perfect health as calculated using the EuroQol 5 Dimension (EQ-5D) outcome measure. ${ }^{4}$ High-quality pain management services can result in a significant reduction in healthcare usage. ${ }^{10}$ This is achieved by teaching patients self-management techniques that are known to reduce disability and distress and improve various measures of physical functions. ${ }^{11-13}$ They have also been shown to facilitate helping patients return to work. ${ }^{14}$

Reciprocally, inadequate inpatient care results in delayed hospital discharge, repeated admissions, high distress in staff and patients and inadequate handover into primary care. ${ }^{15}$ Some patients with complex pain never make it to specialist pain outpatient services because the pain, or potentially related medical condition, repeatedly brings them back into the acute hospital where expert pain multidisciplinary team (MDT) provision is not available.

To compound the above problems, pain is an underfunded specialty with scarce resources. ${ }^{4}$ It is accepted that best practice is to treat patients with chronic pain with an MDT without delay ${ }^{16}$ and that treating this group with expert best practice as early as possible can make significant savings in all areas of healthcare expenditure, not just pain. ${ }^{10}$ However, this knowledge does not currently translate into practice in England where many sufferers have no access at all to specialist services. ${ }^{4}$

\section{THE INTERVENTION}

Inpatients with complex pain will be referred by the medical staff for review by the multidisciplinary complex pain team (CPT). The CPT consists of specialist pain doctors, specialist nurses, physiotherapists and psychologists. Referrals will be considered for patients who fulfil any of the following characteristics which were decided on by local expert opinion and known risk factors for chronicity in the context of long-term pain ${ }^{17} 18$ :

1. Multiple repeat attendances to accident and emergency (A\&E) or general practitioner (GP) for pain-related issues.

2. High opiate use greater than $180 \mathrm{mg}$ oral morphine equivalent per day (British Pain society threshold for high opiate use) ${ }^{19}$

3. No ongoing active management for acute medical or surgical conditions.
4. Non-malignant diagnosis or malignant diagnosis but not appropriate for palliative care input.

5. Ongoing pain for more than 6 months (for inclusion into economic arm so that the six month pre and post intervention comparison is fair).

If the CPT agrees that the patient meets the inclusion criteria above, they will then undergo an immediate and tailored treatment plan with ongoing and regular evaluation. The actual interventions offered by the MDT will not differ from currently accepted best practice and will include the optimisation of pharmacological regimens, cognitivebehavioural therapy, as well as self-management and distraction techniques. The difference is that they will be delivered in the inpatient setting and intensively over a short period. Typically, patients requiring specialist pain interventions would have to wait for an outpatient appointment and furthermore subsequent consultations and interventions would be widely spaced due to demand far outstripping supply with regard to access.

Interventions will be individualised with time spent with the most appropriate health professional on the ward. There will be a large spectrum of clinical input and we anticipate benefit from regular consultations several times per week, as deemed appropriate, when compared with the far greater lengths of time patients are obliged to wait in between outpatient appointments. The CPT will provide an enduring point of contact and allow for efficient and expert communication between the patient, primary care and specialist teams who may be treating the patient for other comorbidities by organising regular MDT meetings. The CPT will also follow-up the patient once discharged with both regular telephone consults and outpatient appointments as deemed appropriate.

This study aims to evaluate a novel MDT inpatient CPT commissioned at a central London teaching hospital using a mixed-methods approach. There is currently no comparative evaluation in the literature nor any evidence that such a service has even been trialled before.

\section{AIM}

To evaluate (using mixed methods) a novel inpatient multidisciplinary CPT.

\section{RESEARCH QUESTIONS}

1. Are core clinical outcomes improved after the inpatient complex pain intervention?

2. How do patients view the CPT when compared with previous experiences of pain services?

3. What are staff perceptions of the impact of the CPT?

4. Is the CPT of overall economic benefit to the health system?

\section{OBJECTIVES}

1. To measure the impact of the CPT on clinical outcomes using validated patient-reported outcome questionnaires (RQ1). 

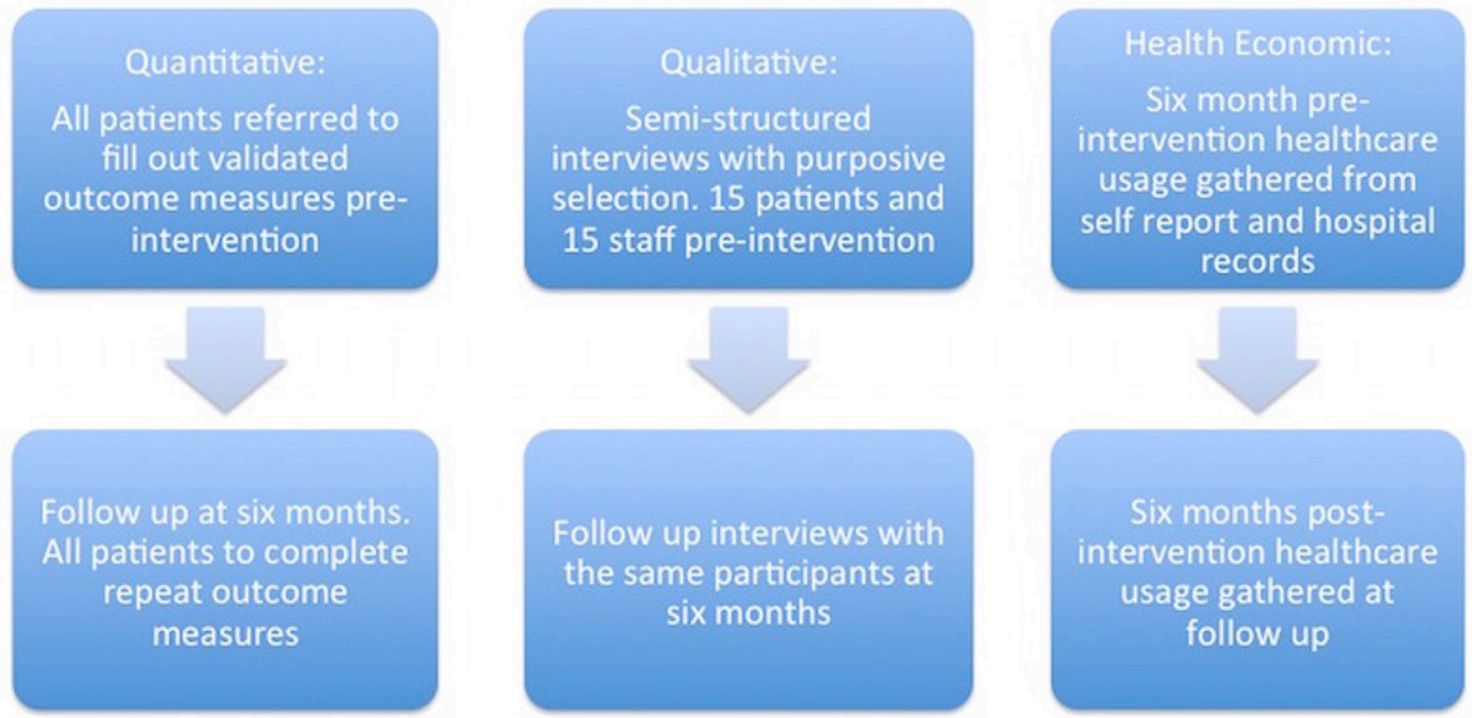

Figure 1 Flow diagram representing different stages of the mixed-methods evaluation.

2. To use semistructured interviews to explore patient experiences of pain management and mechanisms by which clinical effectiveness may have been achieved (or not) (RQ2).

3. To use semistructured interviews to explore staff perceptions of the impact of the CPT (RQ3).

4. To compare healthcare costs for patients with complex pain before and after care delivered by the CPT (RQ4).

\section{METHODS}

\section{Study design}

This is a prospective, observational, single-centre cohort study, which will include all patients receiving the CPT intervention. Patients will be recruited over a 1-year period, and all patients will be followed up at a 6-month interval from the date of admission to the service. From a pilot study at the same London teaching hospital, we estimate that there will be approximately two eligible referrals per week meaning a total study population in the region of 100. Mixed methods will be used to determine the clinical effectiveness of the intervention and explore patient and staff attitudes to the service (see figure 1). Quantitative clinical outcomes and health economic data will be recorded for all eligible participants and 30 preintervention and 30 postintervention semistructured interviews will be conducted with equal numbers of patients and staff.

\section{INTERVIEWS WITH STAFF AND PATIENTS}

Interviews will be semistructured using predetermined topic guides and are expected to last between 15 and 30 min. After consent, the interviews will be recorded using an encrypted Dictaphone and performed in the quietest surroundings available on a ward or preferably in a side room.
Inclusion criteria for interviews for patients accepted to CPT service:

1. Completion of inpatient CPT intervention including completion of all health outcome questionnaires.

2. Capacity to provide written informed consent.

3. Ongoing pain for 6 months or more so as to provide a comparable historic economic comparison.

Exclusion criteria for interviews for patients accepted to $\mathrm{CPT}$ service:

1. Unable to comprehend written and spoken English

2. Lack of capacity to consent.

\section{STAFF INTERVIEWS}

Inclusion criteria:

1. Permanent member of University College London Hospitals (UCLH) staff

2. Regular work on a ward on which the inpatient CPT will operate.

Exclusion criteria:

1. Temporary staff

2. Permanent staff having worked at UCLH for less than 1 year

3. Permanent staff less than 1 year after qualification.

\section{Sampling}

Non-proportional quota sampling will be used to ensure that a representative cross section of age, diagnosis and

Table 1 Sampling brief to show intended number of interviews with patients

\begin{tabular}{lcllll}
\hline & \multicolumn{3}{l}{ No of interviews } & \\
\cline { 2 - 5 } Research stage & Abdominal & Urology & Orthopaedic & Total \\
\hline Preintervention & 9 & 4 & 2 & 15 \\
Postintervention & 9 & 4 & 2 & 15 \\
Total & 18 & 8 & 4 & 30 \\
\hline
\end{tabular}


Table 2 Sampling brief to show intended number of interviews with staff members

\begin{tabular}{llllll}
\hline & \multicolumn{3}{l}{ No of interviews } & & \\
\cline { 2 - 5 } Research stage & $\begin{array}{l}\text { Acute pain } \\
\text { nurses }\end{array}$ & CPT & $\begin{array}{l}\text { Ward } \\
\text { nurses }\end{array}$ & Doctors & Total \\
\hline Preintervention & 2 & 1 & 6 & 6 & 15 \\
Postintervention & 2 & 1 & 6 & 6 & 15 \\
Total & 4 & 2 & 12 & 12 & 30 \\
\hline
\end{tabular}

CPT, complex pain team.

gender is adequately represented, see table 1 below. As per our inclusion criteria, participants must be fluent in both written and spoken English as the study materials are all in this language and the patient will need to be proficient in order to participate in the interview. The interviews will include equal representation of male and female patients as well as younger and older patients (defined as those below the age of 50 years).

The interview population for the staff cohort will be selected using purposive sampling from those willing to take part. Table 2 below shows a sampling brief of those staff members that we intend to interview to provide us with the most informative and cross-sectional data. Interviews will be carried out with nurses as this group of professionals will spend the most time on the ward managing complex pain patients, but variation will be sought in terms of different bands. Interviews will also be conducted with doctors of various levels of seniority and specialities including gastroenterology, haematology and pain.

\section{Recruitment}

Fifteen patients will be recruited for interviews before the intervention and 6 months after the intervention. The researcher will aim to interview the same patients on both occasions. Appropriate patients will be identified after referral, based on the sampling brief shown in table 1, but before any clinical intervention has taken place. At this juncture, a researcher will check with members of the patient's clinical team to see if they can be approached. If the patient agrees, the researcher will approach the patient and provide them with a participant information sheet further detailing the purpose of the interviews. The patient will be given time to read the information sheet and ask the researcher questions. It will be made clear to participants that they are under no obligation to take part in the interviews and their treatment with the CPT will in no way be effected by refusal. If the patient agrees to take part, they will be asked to sign a consent form.

Fifteen members of staff involved with, or clinically effected by, the CPT will be recruited for interviews preintervention and postintervention. The focus will be on wards with historically the most complex pain patients. Staff members will be contacted by the researcher to enquire as to whether they would like to take part in the study. If agreeable, the researcher will give them a participant information sheet and allow them time to ask questions about the study. If they agree to take part in the study, they will be asked to sign a consent form before the interview.

\section{Data collection}

The semistructured interviews broadly contain ten different themes as can be seen in table 3 below. The preintervention topic guides for both patients and staff aim to determine shortfalls that exist in the current system and seek to determine what these two groups would most like improved. These questions were developed with the help of expert advice from the multidisciplinary pain team with the added knowledge that additional themes would evolve naturally during the course of the semistructured interviews.

The postintervention questions seek to determine the impact that the CPT had on both patients and staff and whether these stakeholder groups have any suggestions about how the team might be able to further improve. Once again questions will be refined by themes emerging from the preintervention data.

\section{OUTCOME MEASURES: QUANTITATIVE DATA COLLECTION}

Quantitative data from health questionnaires and prescription medications will be collected from all participants after referral to the CPT but before any intervention and again at 6-month follow-up for comparison. The same researcher will be responsible for all patient contact regarding data collection. There is little consensus regarding which outcome measures should be used to assess a complex pain service. ${ }^{20}$ Physical, emotional and social domains are important although there is no agreement on exactly which outcome measures should be used to represent these. ${ }^{20} 21$ The following health questionnaires were chosen informed by extensive local expert discussion and the latter three key domains. It is hoped that this diverse and comprehensive selection will demonstrate improvements in the various biopsychosocial spheres that are thought to have relevance to long-standing pain as well as showing clinical and functional gains.

- EuroQol-5 Dimension- 5 Level (EQ-5D-5L)-a measure of generic health status developed in Europe. It is a non-disease-specific, standardised and widely used measure of health-related quality of life.

- 12-Item Short Form Survey (SF-12) — another measure of health-related quality of life that is non-disease-specific developed in Northern America.

- Brief Pain Inventory-a questionnaire used to measure the intensity of pain and how it interferes with daily life. Although originally designed to measure cancer pain it has since also been validated in the context of chronic non-malignant pain. ${ }^{22}$

- Patient Health Questionnaire 9-a measure of depression that was designed for use in primary care that is both sensitive and specific. ${ }^{23}$ It has since been validated in the context of many specific conditions including chronic pain. ${ }^{24}$ 
Table 3 Topic schedule for the patient and staff, preintervention and postintervention interviews

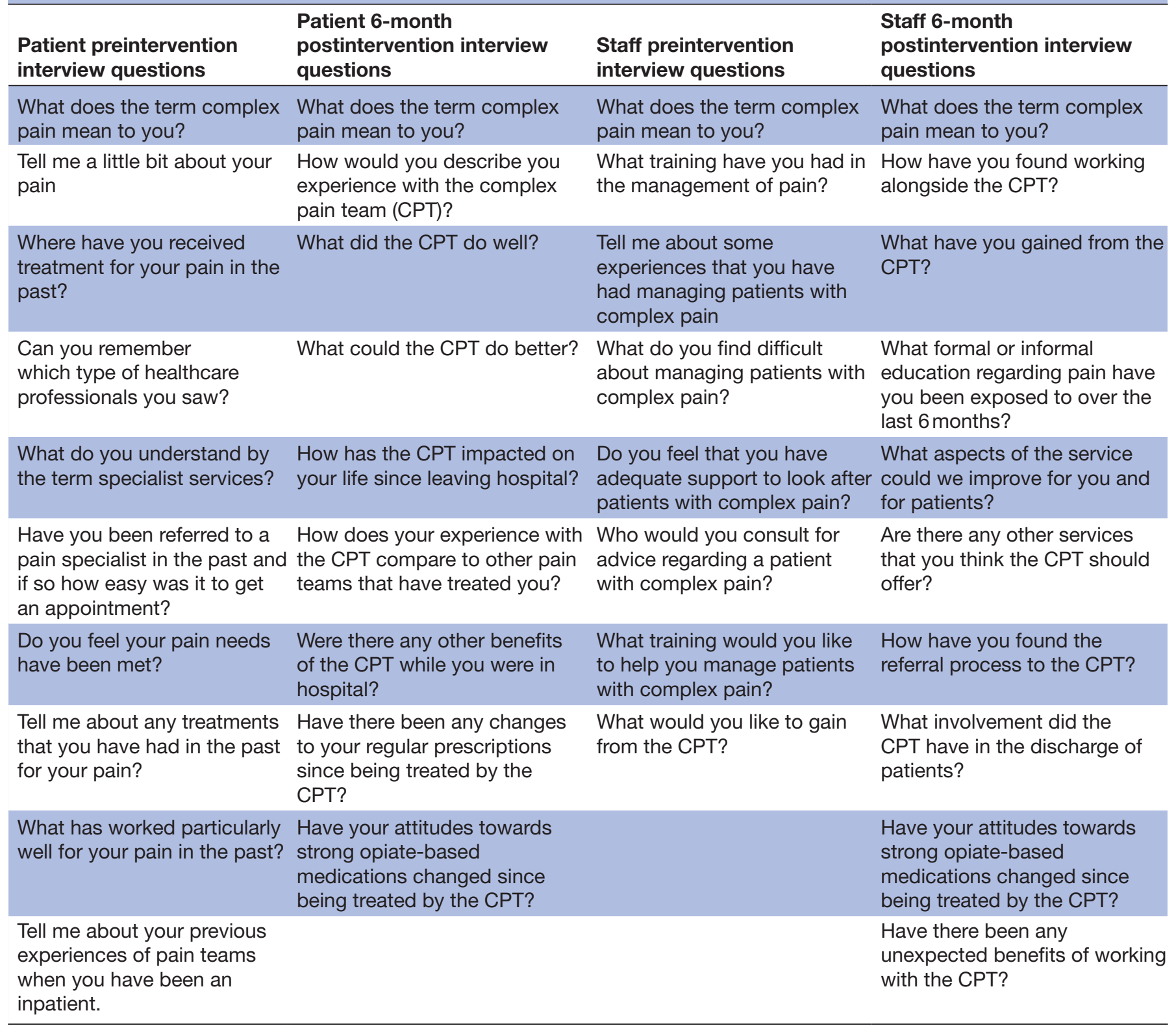

- Pain Catastrophising Scale-a measure of catastrophic thinking, higher levels of which can result in a more intense experience of pain. ${ }^{25}$

- Pain Self-Efficacy Score-a measure of one's overall confidence in their ability to deal with the pain and the impact it has on their day-to-day functionality. The ability to successfully deal with stresses and symptoms is associated with better outcomes. ${ }^{26}$

- Health economic data collection-the researcher will collect health economic data, in the form of self-reported healthcare usage, at the same time that the participant completes the clinical outcome measures preintervention and again at 6 months postintervention. Additionally hospital electronic records will be accessed and used to document care given in the specific trust.

\section{DATA COLLECTION FOR FOLLOW-UP}

Research personnel will follow-up all participants at 6 months. For patients, this will opportunistically coincide with existing outpatient appointments already scheduled at the trust where quantitative outcome forms will be completed and a researcher will enquire as to healthcare usage during the 6-month follow-up period. Follow-up interviews will also be carried out at this juncture for those included in the qualitative analysis. For those that are unable to come to the hospital or do not have any outpatient appointments scheduled this follow-up procedure will be performed over the telephone.

Staff participants in the qualitative analysis will be interviewed at a time of mutual convenience at the hospital after the stated interval of 6 months. 


\section{PARTICIPANT ELIGIBILITY CRITERIA AND RECRUITMENT}

All patients referred to and accepted for treatment by the CPT will be recruited into the quantitative clinical and health economic arms of the study. Baseline patient-reported outcomes questionnaires will be administered to patients preintervention on the ward and collected by a researcher who at the same time will have a detailed discussion regarding exact healthcare usage during the preceding 6 months. The latter conversation will enquire after hospital admissions, the diagnoses responsible and length of stay. GP, A\&E and outpatient attendances as well as district nurse home visits will be discussed, and any operations, investigations and procedures will be recorded.

\section{DATA ANALYSIS}

\section{Qualitative analysis}

In the first instance, the research team will use the interviews to explore patient and staff satisfaction. The latter is detailed by the Initiative on Methods, Measurement and Pain Assessment in Clinical Trials, as one of six important outcomes measures when evaluating a pain intervention. Direct immediate feedback from these interviews will allow the service to evolve in a very responsive way as issues are identified and discussed at monthly progress meetings with the team.

All qualitative data will be formally analysed using framework analysis, using the research questions outlined above as the main thematic categories, while also being sensitive to additional themes that might emerge from the data.

The topic guide used to explore the above will enquire about the staffs' confidence and attitudes when interacting with patients suffering with complex pain and in particular what makes it difficult; the attitudes of staff and the perceived attitudes of patients' to strong analgesic drugs; the level of support that staff feel that they have regarding complex pain and the pathways around advice and referral and if they would like more help; education in matters pertaining to complex pain and how staff think both themselves and patients might benefit from the novel complex pain service. The 6-month follow-up interviews will seek to identify any changes in attitudes and or practice in the above areas that may have resulted from the presence of the CPT.

Preintervention interviews with patients will focus on areas of unmet need and trying to establish a deeper understanding of some of the barriers to the effective management of complex pain in the hospital environment. Patients' attitudes and beliefs surrounding strong analgesics will also be discussed. The 6-month follow-up interviews will concentrate on differences patients noticed between the CPT and encounters with previous pain teams particularly with regard to personnel, communication and understanding. The researcher will ask whether any particular aspect of the CPT intervention was of exceptional benefit and if that translated into any material differences postdischarge from hospital. Attitudes towards strong, particularly opiate class medications will again be revisited and the researcher will enquire after any changes or improvements to the service that could be suggested.

Advice has been sought from researchers with experience in qualitative methods from the UCLH-Embedded Research Team.

\section{Quantitative analysis}

The results from the outcome measures listed above will be analysed using appropriate two-tailed tests to assess for statistically significant differences between the preintervention and postintervention groups. Given the limitations regarding small population size multivariate analysis will likely not be possible.

\section{Health economic analysis}

The target population of the economic analysis will be all inpatients over the age of 18 years, in which the CPT intervened in their care. In the absence of a formal control group two other comparisons will be made. In the first instance trial, CPT inpatients will act as their own controls and the healthcare costs incurred for each patient in the 6 months preintervention will be compared with those incurred in the 6 months post. This aims to substantiate existing evidence that pain management programmes reduce healthcare usage and hence costs. ${ }^{10}$ The second comparator group will rely on a cohort of historical patients from the same hospital treated in the year prior to the inception of the CPT by the pre-existing non-multidisciplinary pain team. Although not formally matched this will represent a similar population regarding the demographics and pathologies of patients that routinely present to the hospital. For this cohort, a similar calculation of 6-month preintervention and postintervention costs will be performed.

The data on hospital usage will be sourced from both hospital records and self-report. Episodes that take place within the trial hospital will be summed directly from electronic records. Additional healthcare usage, in primary care and other NHS Trusts, will be elicited from patients by a researcher at the same time as filling in clinical outcome forms. Patients will be asked about GP visits, other hospital admissions and outpatient appointments as well as A\&E attendances during the 6 months prior. Exact treatment costs will be sourced from publically available NHS reference data.

The above data will allow two primary analyses to be performed. First, the CPT patients will act as their own controls and healthcare spending in the 6 months prior to referral will be compared with the 6 months post in a direct cost comparison. The second analysis will compare the difference in costs between the CPT patients and the matched cohort. This comparison will allow relative savings to be assessed using a difference of differences analysis of both pregroups and postgroups. Balancing costs of staff provision regarding the CPT and 
pre-existing pain team will be calculated and taken into account.

Additionally, changes in repeat prescriptions as issued by the participant's GP will be recorded at 6-month follow-up and compared regarding costs with preintervention medications. Drug costs will be sourced using the British National Formulary.

There are various limitations and assumptions that must be declared using this methodology. Six-month windows were pragmatically chosen as the longest period possible given the availability of research time and personnel, although a far longer time period would ideally be employed to robustly demonstrate change. Hospital records are accurate although there will doubtless be errors in patients self-reporting of healthcare use when asked about the previous 6 months. Applying for access to local Clinical Commissioning Group (CCG) patientlevel data or Hospital Episode Statistics was not possible on account of the lag time in acquiring said data and the very small sample size of this study's population.

At an absolute minimum the health economic analysis hopes to demonstrate that the cost of providing the service is offset by a subsequent reduction in healthcare usage. This would provide a strong case to commission the service on a permanent basis as one that provides best practice and yet is cost neutral. The authors hope, however, that the savings accrued downstream considerably outweigh the costs of the CPT.

\section{COMBINING RESEARCH METHODS}

Research Methods will be combined throughout the duration of this study. Interview topics will take into account data gathered from the quantitative clinical outcomes and health usage to inform additional pertinent avenues of questioning. Eventually, results from both the qualitative and quantitative aspects of this study will be interpreted in unison to enrich understanding of why given outcomes were achieved, or not, and allow for a more informed evolution of the service.

\section{STUDY STATUS}

The study will finish recruiting patients to all arms of the analysis at the end of January 2017 and follow-up will be completed by November 2017. Although the research team aims to follow-up all participants at 6 months, it is often difficult to arrange. A window of up to 9 months is considered acceptable to ensure the inclusion of as many results as possible.

\section{ETHICS AND DISSEMINATION}

The protocol for this study was formally reviewed by the Joint Research Office at University College London/ UCLH and by the Governance Lead at University College Hospital and was it deemed to represent a service evaluation.
On completion of the research, we intend to report the results via publication in peer-reviewed scientific journals and in the form of conference presentations.

Information will be included in the patient information sheet detailing how they can gain access to the study results when available.

Contributors LM, CV-P and VJW helped with the development of qualitative evaluation and semistructured interview schedules and editing of protocol. NS, SM helped with the development of economic study design and editing of protocol. AW, $\mathrm{NC}$ and RM helped with the development of quantitative clinical study design and editing of protocol.

Funding CV-P, SM, NS and VJW were in part supported by the National Institute for Health Research (NIHR) Collaboration for Leadership in Applied Health Research and Care North Thames at Barts Health National Health Service (NHS) Trust.

Disclaimer The views expressed are those of the authors and not necessarily those of the NHS, the NIHR or the Department of Health.

Competing interests None declared.

Patient consent Obtained.

Provenance and peer review Not commissioned; externally peer reviewed.

Open Access This is an Open Access article distributed in accordance with the Creative Commons Attribution Non Commercial (CC BY-NC 4.0) license, which permits others to distribute, remix, adapt, build upon this work non-commercially, and license their derivative works on different terms, provided the original work is properly cited and the use is non-commercial. See: http://creativecommons.org/ licenses/by-nc/4.0/

(c) Article author(s) (or their employer(s) unless otherwise stated in the text of the article) 2018. All rights reserved. No commercial use is permitted unless otherwise expressly granted.

\section{REFERENCES}

1. GOV.UK. Department of Health and Social Care. http://www.dh. gov.uk/prod_consum_dh/groups/dh_digitalassets/documents/ digitalasset/dh_096233.pdf.

2. Belsey J. Primary care workload in the management of chronic pain. A retrospective cohort study using a GP database to identify resource implications for UK primary care. J Med Economics 2002;5:39-50.

3. Health and Social Care Information Centre. Health survey for England 2011 (HSCIC, Leeds). 2012 https://catalogue.ic.nhs.uk/publications/ public-health/surveys/heal-surv-eng 2011/HSE2011-Ch9-ChronicPain.pdf

4. British Pain Society. National pain audit final report 2010-2012 (British Pain Society, London). 2012 https://www.britishpainsociety. org/static/uploads/resources/files/members_articles_npa_2012_1.pdf (accessed 29 Jan 2018).

5. Fayaz A, Croft P, Langford RM, et al. Prevalence of chronic pain in the UK: a systematic review and meta-analysis of population studies. BMJ Open 2016;6:e010364.

6. Blyth FM, March LM, Brnabic AJ, et al. Chronic pain in Australia: a prevalence study. Pain 2001;89:127-34.

7. Gureje O, Von Korff M, Simon GE, et al. Persistent pain and wellbeing: a World Health Organization Study in Primary Care. JAMA 1998;280:145-51.

8. Verhaak PF, Kerssens JJ, Dekker J, et al. Prevalence of chronic benign pain disorder among adults: a review of the literature. Pain 1998;77:231-9.

9. Gatchel RJ. Comorbidity of chronic pain and mental health disorders: the biopsychosocial perspective. Am Psychol 2004;59:795-805.

10. Clare A, Andiappan M, MacNeil S, et al. Can a pain management programme approach reduce healthcare use? Stopping the revolving door. Br J Pain 2013;7:124-9.

11. van Tulder MW, Ostelo R, Vlaeyen JW, et al. Behavioral treatment for chronic low back pain: a systematic review within the framework of the Cochrane Back Review Group. Spine 2000;25:2688-99.

12. Guzmán J, Esmail R, Karjalainen K, et al. Multidisciplinary rehabilitation for chronic low back pain: systematic review. BMJ 2001;322:1511-6.

13. Morley S, Eccleston C, Williams A. Systematic review and metaanalysis of randomised controlled trials of cognitive behaviour 
therapy and behaviour therapy for chronic pain in adults, excluding headache. Pain 1999;80:1-13.

14. Watson PJ, Booker CK, Moores $\mathrm{L}$, et al. Returning the chronically unemployed with low back pain to employment. Eur J Pain 2004;8:359-69.

15. Societal Impact of Pain (SIP). SIP proposal for action: counting the cost of pain: the whole system impact of poor pain management in the UK, 2013. http://www.sip-platform.eu

16. Patrick LE, Altmaier EM, Found EM. Long-term outcomes in multidisciplinary treatment of chronic low back pain: results of a 13year follow-up. Spine 2004;29:850.

17. Mallen CD, Peat G, Thomas E, et al. Prognostic factors for musculoskeletal pain in primary care: a systematic review. Br J Gen Pract 2007;57:655-61.

18. Linton SJ. A review of psychological risk factors in back and neck pain. Spine 2000;25:1148-56.

19. https://www.britishpainsociety.org/static/uploads/resources/files/ book_opioid_main.pdf
20. Deckert S, Kaiser U, Kopkow C, et al. A systematic review of the outcomes reported in multimodal pain therapy for chronic pain. Eur $J$ Pain 2016;20:51-63.

21. Dworkin RH, Turk DC, Farrar JT, et al. Core outcome measures for chronic pain clinical trials: IMMPACT recommendations. Pain 2005;113:9-19.

22. Tan G, Jensen MP, Thornby JI, et al. Validation of the brief pain inventory for chronic nonmalignant pain. $J$ Pain 2004;5:133-7.

23. Kroenke K, Spitzer RL, Williams JB. The PHQ-9: validity of a brief depression severity measure. J Gen Intern Med 2001:16:606-13.

24. Choi Y, Mayer TG, Williams MJ, et al. What is the best screening test for depression in chronic spinal pain patients? Spine $J$ 2014;14:1175-82.

25. Leung L. Pain catastrophizing: an updated review Indian. J Psychol Med 2012;34:204-17.

26. Jackson T, Wang Y, Wang Y, et al. Self-efficacy and chronic pain outcomes: a meta-analytic review. J Pain 2014;15:800-14. 